\title{
Effects of nonidealities in switching frequency modulation techniques: application to Coupled Interleaved Multicellular Parallel converters.
}

\author{
Juan Mon ${ }^{1}$, David Gonzalez ${ }^{1}$, Lluís Ferrer-Arnau ${ }^{1}$, Denis Labrousse ${ }^{2}$, François Costa $^{2}$ \\ ${ }^{1}$ Departament Enginyeria Electrònica \\ Universitat Politècnica de Catalunya \\ Terrassa, Spain \\ Tel.: +34937398936 \\ Fax: +34937398016 \\ ${ }^{2}$ SATIE, ENS Cachan, CNRS, UniverSud, \\ 61 av. du Président Wilson, \\ 94320 Cachan, France \\ Tel.: +33147402108 \\ Fax: +33147402199 \\ E-Mail: juan.mon@upc.edu \\ E-Mail: denis.labrousse@satie.ens-cachan.fr
}

\section{Acknowledgements}

This research is supported by the Ministerio de Ciencia e Innovación in the frame of the project TEC2011-25076.

\section{Keywords}

«EMC/EMI», «Interleaved converters », «Modulation strategy », «Pulse Width Modulation (PWM)».

\begin{abstract}
This paper explores the application of the Variable Delay Frequency Modulation (VDFM) to an Interleaved Multicellular Parallel converter. VDFM is a modulation technique specially intended to reduce conducted EMI generated by parallel converters. This technique combines interleaving and frequency modulation in such a way that provides the best attenuation. On the other hand, Coupled Interleaved Multicelular Parallel Converters (CIMPC) needs a perfect current sharing among phases. In this paper we consider the effect of VDFM nonidealities on the EMI attenuation in order to consider the application of this technique to CIMPC.
\end{abstract}

\section{Introduction}

It has been demonstrated that switching frequency modulation is an effective way to reduce conducted EMI produced by switched power converters. Recently, the Variable Delay with switching Frequency Modulation (VDFM) has been presented [1-3]. This technique, which combines interleaving with frequency modulation, is specially intended for converters in parallel topology and shows good results in terms of conducted disturbances attenuation [4]. Moreover, VDFM provides a good tradeoff between EMI attenuation and undesirable side effects, such as output voltage ripple increase.

On the other hand, the Coupled Interleaved Multicellular Parallel Converter (CIMPC) shows advantages in terms of system integration, power density and dynamic performance $[5,6]$. For these reasons, CIMPC topology is widely used in applications where outstanding dynamic performance is required, such in Voltage Regulator Modules (VRM) [7]. However, in this kind of converters, which are operated with interleaving, an accurate control and modulation are required in order to ensure a proper current balancing among each single switching cell of the converter [8]. This situation is more relevant in battery charging application, where a current mode control is necessary in order to adjust the battery charging current [9]. In a practical implementation, some constructive differences in switch drivers, stray impedances, etc, are unavoidable. These differences result to an unequal current distribution among phases of the parallel converter. In order to adjust the current distribution, it is necessary to vary the duty cycle in each phase from the ideal duty cycle. The dispersion in duty cycle 
values is identified as the most important parameter in attenuation degradation when pure interleaving is applied [7, 10]. This paper explores the feasibility of application of VDFM to CIMPC. We pay special attention to the effect of the duty cycle dispersion due to non-idealities of the system on the EMI attenuation.

This paper is organized as follows. First of all, the basics of VDFM and some experimental results of attenuation as well are presented. In the next section, the origin of duty cycle deviation is analysed and some preliminary simulation results considering dispersion in the duty cycle are evaluated. In order to verify the effect of the duty cycle deviation on the conducted EMI, the EMI spectrum for different duty cycle deviation in a four phases parallel buck converter is compared. Finally, the main conclusions are summarized.

\section{VDFM theory and results}

Figure 1a illustrates general switching patterns for a multichannel modulator, where $i$ notes each phases $(i=1,2, . ., N), \alpha_{i}$ is the delay among switching patterns, $q_{i}(t)$, and $\varepsilon_{k, i}$ is the pulse position delay inside the $k$-th switching cycle. Notice that each switching pattern has a period equal to the period of the modulation profile, $T_{m}$, and contains $L$ switching cycles (1). There are several possibilities of combination interleaving and spread spectrum modulation in multiconverter arrangement [4]. The VDFM, which pattern is shown in Fig. 1b, combines the interleaving and switching frequency modulation techniques.

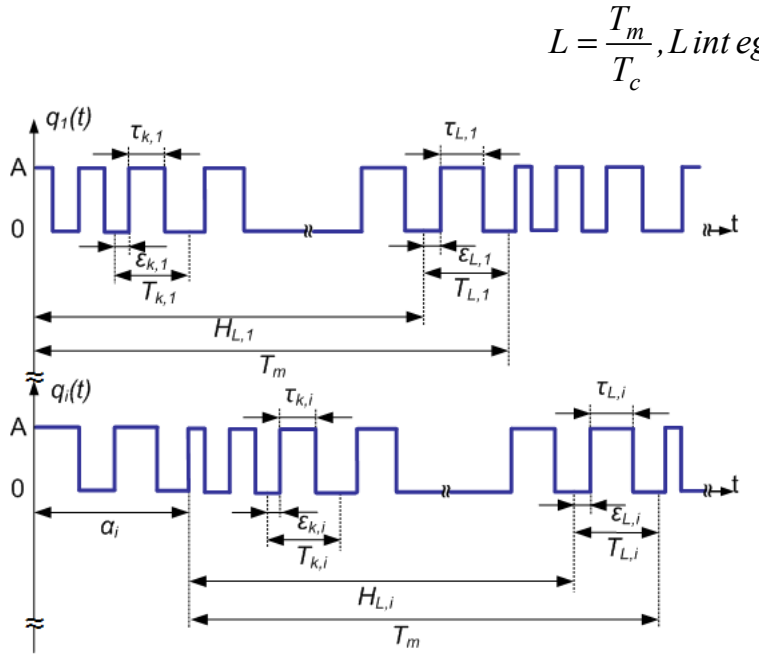

(a)

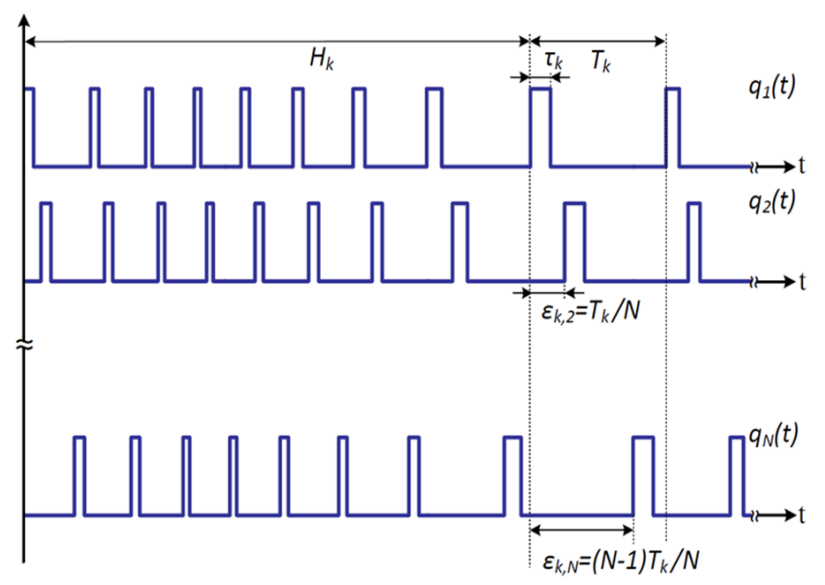

(b)

Fig. 1. Switching pattern. (a) General switching pattern for a multicellular converter. (b) VDFM switching pattern.

In VDFM there is any delay among switching patterns $\left(\alpha_{i}=0\right)$ but a variable pulse delay of pulse position in each switching period, $\varepsilon_{k, i}$, is introduced depending on the instantaneous switching period according to the expression (2),

$$
\varepsilon_{k, i}=\frac{T_{k}}{N} \cdot(i-1)
$$

The instantaneous switching period, $T_{k}$, when a periodic modulation profile is used, can be approximated to (3),

$$
T_{k}=T_{c}+\Delta T_{k} \approx \frac{1}{f_{c}+\Delta f_{c} V_{m}\left(H_{k}\right)}
$$


where $f_{c}$ is the central switching frequency, $\Delta f_{c}$ is the maximum frequency deviation and $H_{k}$ is the starting time of the $k$-th switching cycle, that is given by (4).

$$
H_{k}=\left\{\begin{array}{cc}
0 & k=1 \\
\sum_{j=1}^{k-1} T_{j} & k>1
\end{array}\right.
$$

The equivalent noise pattern in frequency domain corresponding to VDFM, $S_{V D F M}(w)$, is expressed by expression (5),

$$
S_{V D F M}(w)=F\left\{\sum_{i=1}^{N} q_{i}(t)\right\}=N A D_{c} \partial(w)+\sum_{n=1}^{\infty}\left(\frac{A}{j \pi n} \cdot \sum_{k=1}^{L}\left[e^{\frac{-j 2 \pi n H_{k}}{T_{m}}}\left(\frac{1-e^{\frac{-j 2 \pi n T_{k}}{T_{m}}}}{1-e^{\frac{-j 2 \pi n T_{k}}{N T_{m}}}}\right)\left(1-e^{\frac{-j 2 m D_{c} T_{k}}{T_{m}}}\right) \partial \partial\left(w-n w_{m}\right)\right)\right.
$$

where $D_{c}$ is the duty cycle established by the controller.

In Fig. 2, the equivalent noise spectrum for interleaving and VDFM is depicted. It is observed a reduction in the harmonics amplitude when the VDFM method is applied, because the energy of each interference harmonics has been distributed into side-band harmonics.

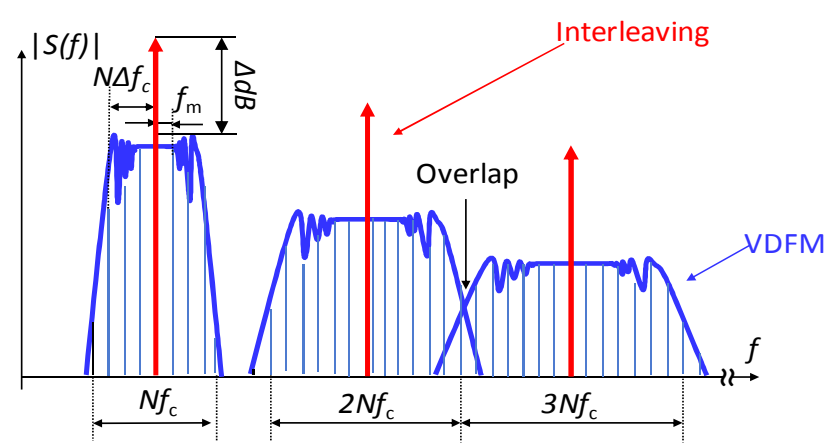

Fig. 2. Spread bands of harmonics in VDFM.

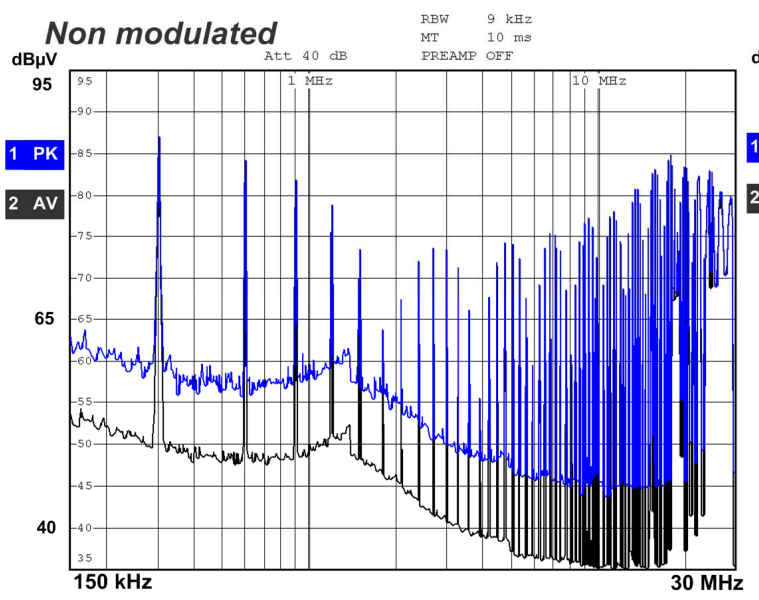

(a)

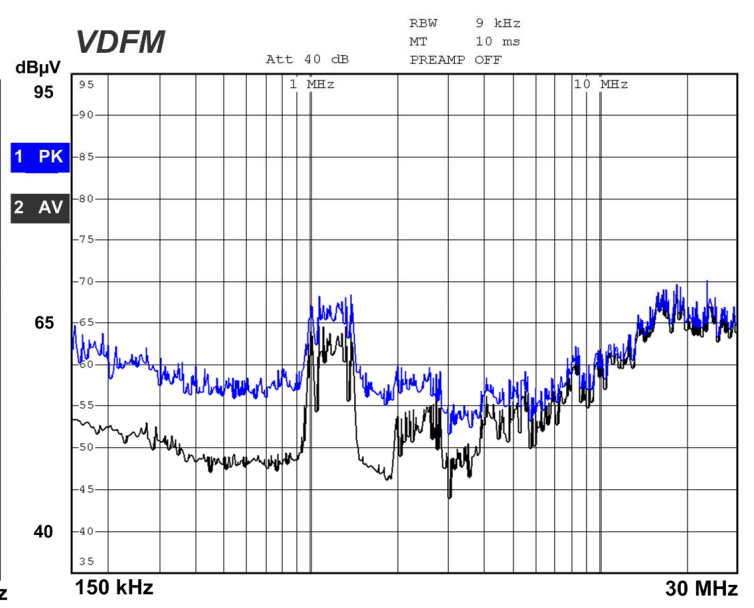

(b)

Fig. 3. Conducted disturbances. (a) Without interleaving neither modulation. (b) VDFM. (Blue trace: Peak detector ; Black trace: Average detector)

VDFM has been experimentally tested in a $4(N=4)$ phases buck converter without current balance algorythm, operated at a central frequency of $f_{c}=300 \mathrm{kHz}$. Figure 3a shows the conducted EMI with the four cells operated without modulation neither interleaving, where the bands multiple of the fundamental frequency are clearly visible. If VDFM is applied, we get the spectrum shown in Fig. 3b. Due to interleaving, only components at $N f_{c}$ remains, that is $1.2 \mathrm{MHz}, 2.4 \mathrm{MHz}$ and so on. The energy 
of the remaining harmonics is spread in sidebands. This effect is clearly visible in Fig. $3 \mathrm{~b}$ around $1.2 \mathrm{MHz}$ and $2.4 \mathrm{MHz}$ as well. At higher frequencies, the overlap among sidebands appears and particular groups of sidebands are no more distinguishable.

\section{Nonidealities of VDFM}

The effect of cancellation of the VDFM technique depends on the variation of the duty cycle among switching patterns. It should be noticed that in VDFM, the duty cycle on each switching cycle is set by the control loop. Duty-cycle mismatch can be caused by different factors. The most relevant are switch driver imperfections and impedance mismatch among converter phases [7, 10]. The effect of driver imperfections, such as rise/fall times, or unequal switching behavior of devices is only noticeable for central switching frequencies in the range of MHz. For this reason, we do not consider this fact in this paper.

Regarding the second reason, mismatch of parasitic impedances among phases produce a current unbalance [7]. Therefore, a duty cycle deviation from the ideal case must be introduced to equally share currents among phases, according to (6),

$$
\Delta I_{L i}=-I_{i} \frac{N-1}{N} \cdot \frac{\Delta R_{i}}{R}=\frac{N-1}{N} \cdot \frac{V_{i n} \cdot \Delta D_{i}}{R}
$$

where subindex $i$ corresponds to each of the $N$ switching patterns, $R$ is the stray "common" impedance, $\Delta R_{i}$ is the deviation of the stray impedance and $\Delta D_{i}$ is the deviation of duty cycle.

Figure 4 illustrates a "worst-case" scenario, where all phases have the same average current, $I_{L, i}$ (we consider there are no difference among these phases in terms of stray impedances), except phase $N$, that has a different average current, $I_{L, N}$. This leads to an average current difference expressed by (7), where $I_{\text {ref }}$ is the current reference.

$$
\Delta I_{L i}=I_{L i}-I_{r e f}
$$

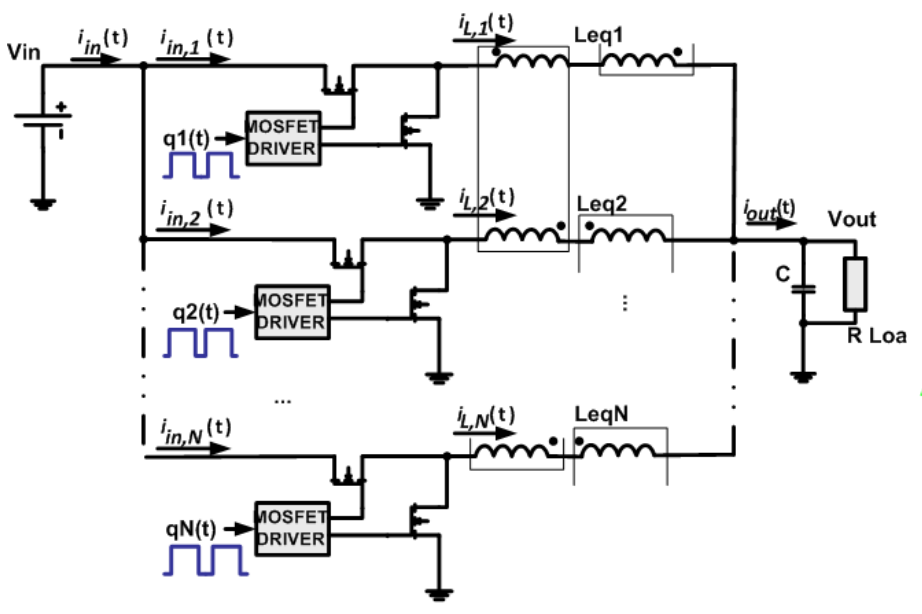

(a)

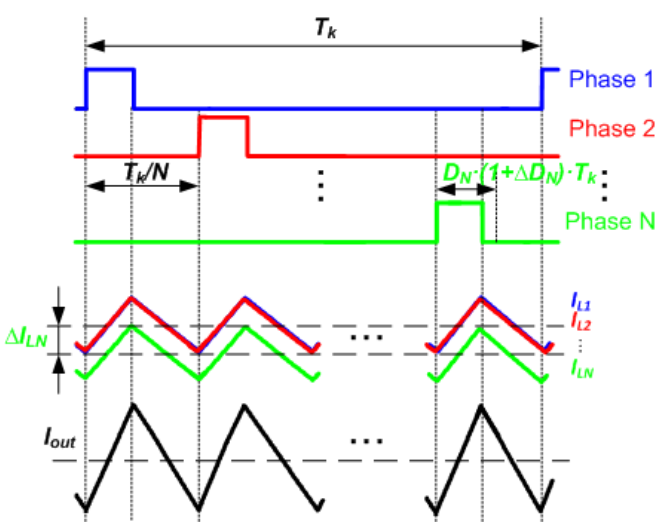

(b)

Fig. 4. $N$ phases buck converter with coupled inductors. (a) Block diagram. (b) Main waveforms.

In order to evaluate the effect of duty cycle deviation, the duty cycle corresponding to switching pattern of phase $N$ has been modified according to (8) and has been normalized respect to $D_{c}$. The other patterns have a duty cycle equal to $D_{c}$.

$$
D_{N}=D_{c} \cdot(1+\Delta D)
$$


The modulation parameters shown in Table I have been used to simulate the effects of the duty cycle deviation for different number of phases, $N$. (Note: these values are the same that have been used to carry out experiments shown in Section II).

TABLE I: Modulation parameters

\begin{tabular}{|c|c|c|c|c|c|}
\hline Number of phases & $f_{c}$ & $\Delta f_{c}$ & Modulation profile & $f_{m}$ & $D_{c}$ \\
\hline 3 & \multirow{2}{*}{$300 \mathrm{kHz}$} & $60 \mathrm{kHz}$ & Triangular & $10 \mathrm{kHz}$ & $13.5 \%$ \\
\hline 4 & & & & & \\
\hline
\end{tabular}

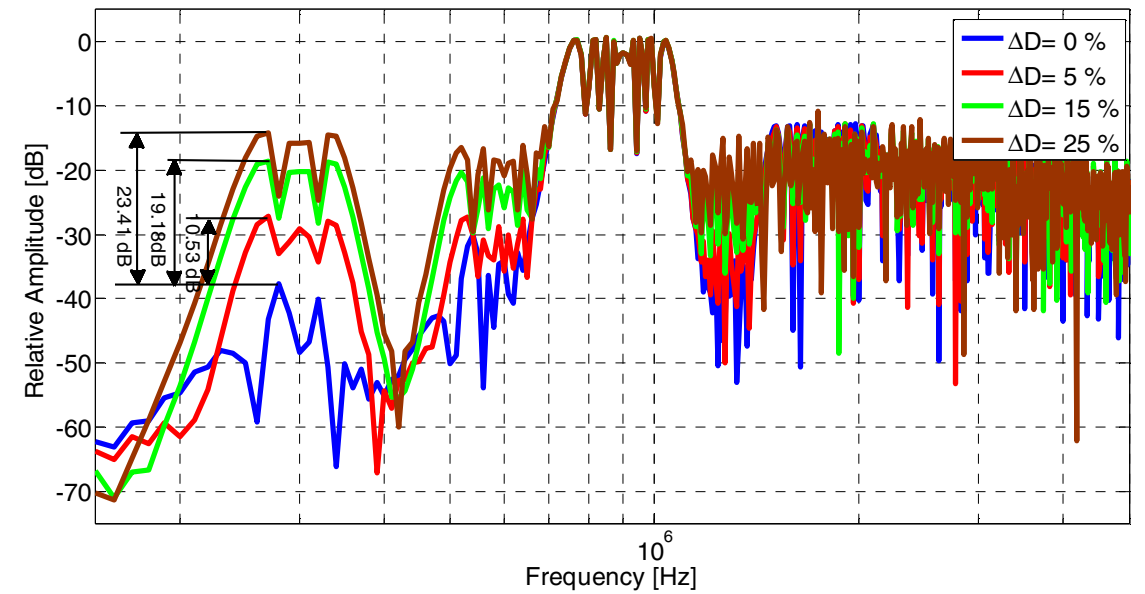

(a)

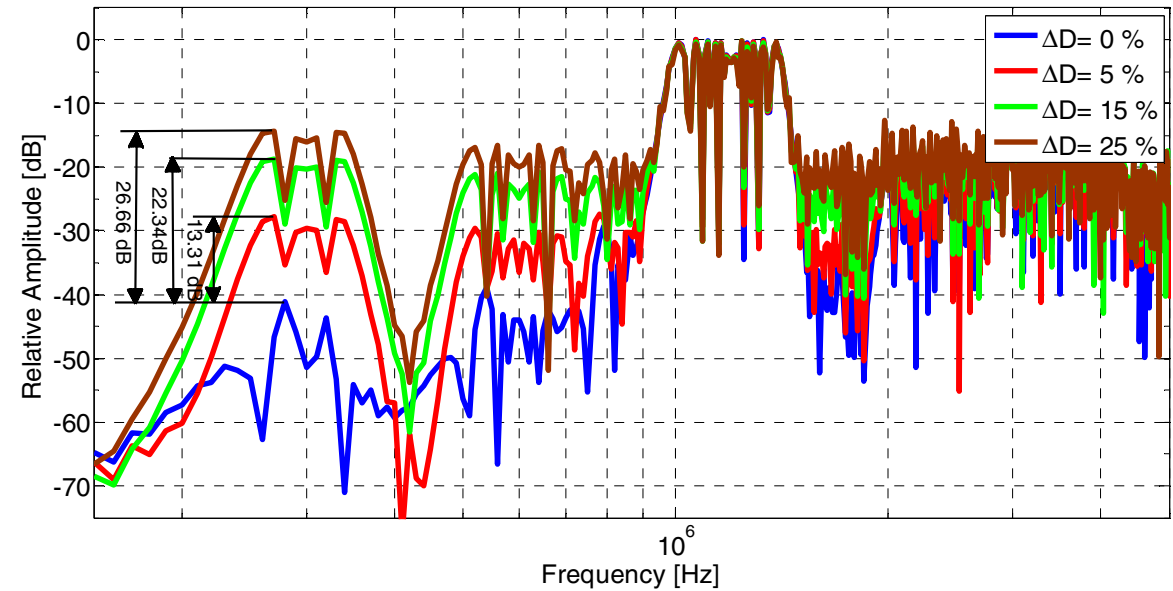

(b)

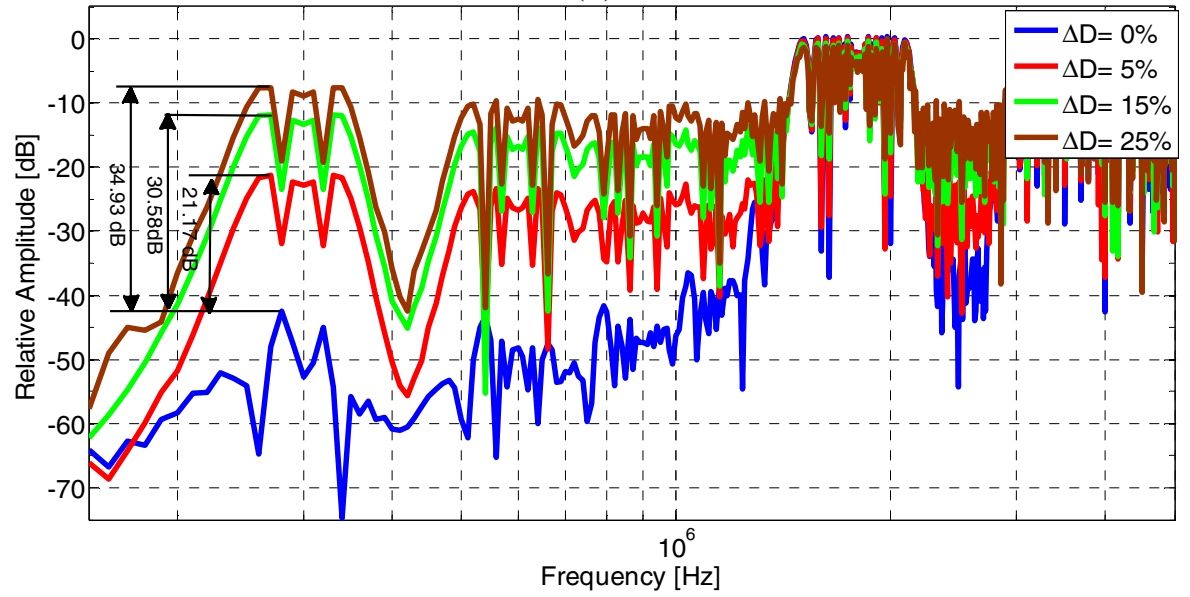

(c)

Fig. 5. Effect of duty cycle deviation on the conducted EMI spectrum. (a) $N=3$. (b) $N=4$. (c) $N=6$. 
Figure 5 shows the effect of the duty cycle deviation for $N=3, N=4$ and $N=6$ in the conducted EMI spectrum. In the ideal case $(\Delta D=0 \%$; blue trace), only sideband harmonics around frequencies multiple of $N f_{c}$ are observed. The effect of duty cycle deviation is highly noticeable for frequencies below $N f_{c}$. With a deviation of $5 \%$, the attenuation degradation is $10 \mathrm{~dB}$ for $N=3,13 \mathrm{~dB}$ for $N=4$ and $21 \mathrm{~dB}$ for $N=6$, respectively. However, any attenuation degradation is noticed around $N f_{c}$. For frequencies above $3 N f_{c}$, overlap among consecutive sidebands appears and fades the effect of duty cycle deviation. In other words, attenuation degradation is almost unnoticeable where overlap appears.

On the other hand, we see that the same absolute value of duty-cycle deviation produces more impact for higher number of phases. For instance, for $N=3$ and a $\Delta D$ of $25 \%$ results in the same loose of attenuation than $N=4$ and $\Delta D$ of $15 \%$ (18.6\% theoreticaly).

\section{Experimental Results}

The influence of duty-cycle deviation on the conducted EMI attenuation have been evaluated in a four phases $(N=4)$ buck converter operated in open loop. The main specifications of the convert are summarized in Table II. The experimental results have been obtained according to the modulation parameters shown in Table I.

TABLE II: Main specifications of multichannel buck converter

\begin{tabular}{|c|c|c|c|c|c|}
\hline $\begin{array}{c}\text { Nominal Input } \\
\text { Voltage }\end{array}$ & $\begin{array}{c}\text { Nominal Output } \\
\text { Voltage }\end{array}$ & Power & $\begin{array}{c}\text { Switching } \\
\text { Frequency }\end{array}$ & $\begin{array}{c}\text { Number of } \\
\text { phases }\end{array}$ & $\mathrm{L}_{\text {eq,i }}$ \\
\hline $12 \mathrm{~V}_{\mathrm{DC}}$ & $1.5 \mathrm{~V}_{\mathrm{DC}}$ & $4 \mathrm{~W}$ & $300 \mathrm{kHz}$ & 4 & $68 \mu \mathrm{H}$ \\
\hline
\end{tabular}

Figures 6, 7 and 8 compare the effect of duty-cycle deviation of $5 \%, 15 \%$ and $25 \%$ to the ideal case, respectively. Experimental results match the simulation predictions. Attenuation degradation is clearly noticed for frequencies below the frequency on which sidebands overlap appears ( $3 \mathrm{MHz}$ in this case). Fore instance, at $f_{c}=300 \mathrm{kHz}$ an attenuation loose of $10 \mathrm{db}, 20 \mathrm{~dB}, 25 \mathrm{~dB}$ is observed (please, noticed that noise floor in Fig. $3 \mathrm{~b}$ is $50 \mathrm{~dB}$ ) for $\Delta D$ equal to $5 \%, 15 \%$ and $20 \%$, respectively. The imperfect cancellation of sidebands due to duty-cycle deviation is visible for frequencies of $2 f_{c}$ and $3 f_{c}$. For frequencies above this value, the effect is barely visible.

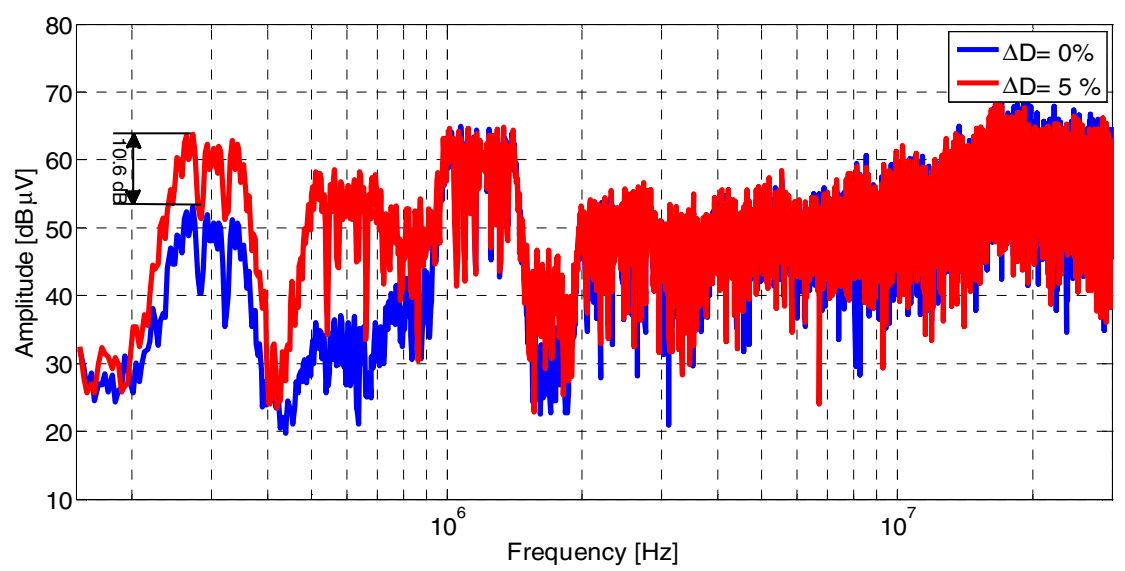

Fig. 6. Conducted EMI for $\Delta D=5 \%$. (Blue trace: $\Delta D=0 \%$; Red trace: $\Delta D=5 \%$ ) 


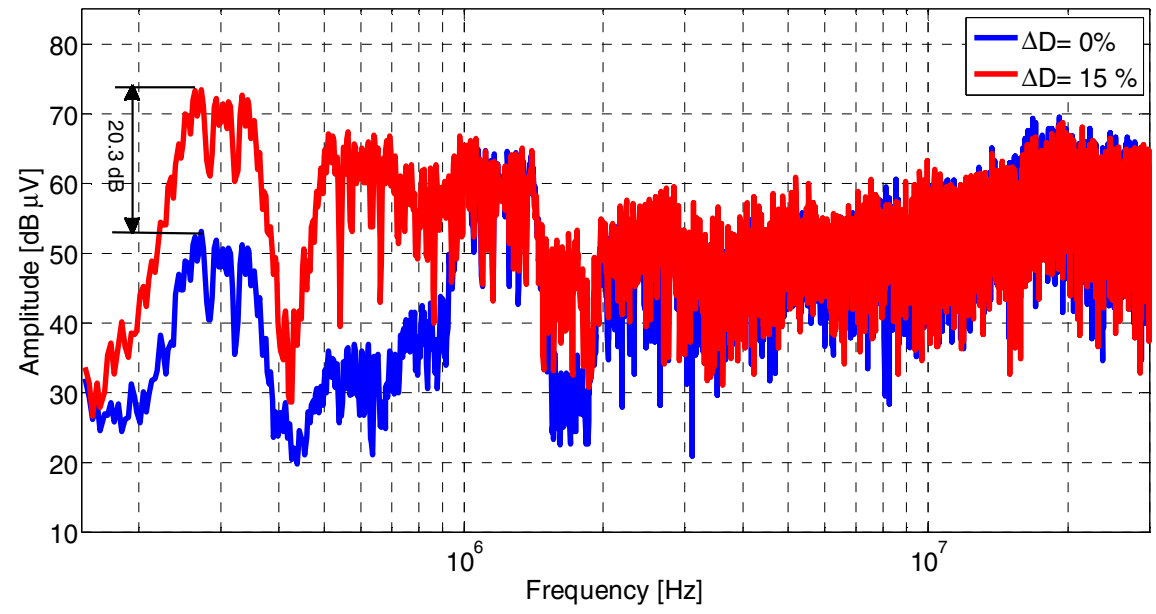

Fig. 7. Conducted EMI for $\Delta D=15 \%$. (Blue trace: $\Delta D=0 \%$;Red trace: $\Delta D=15 \%$ )

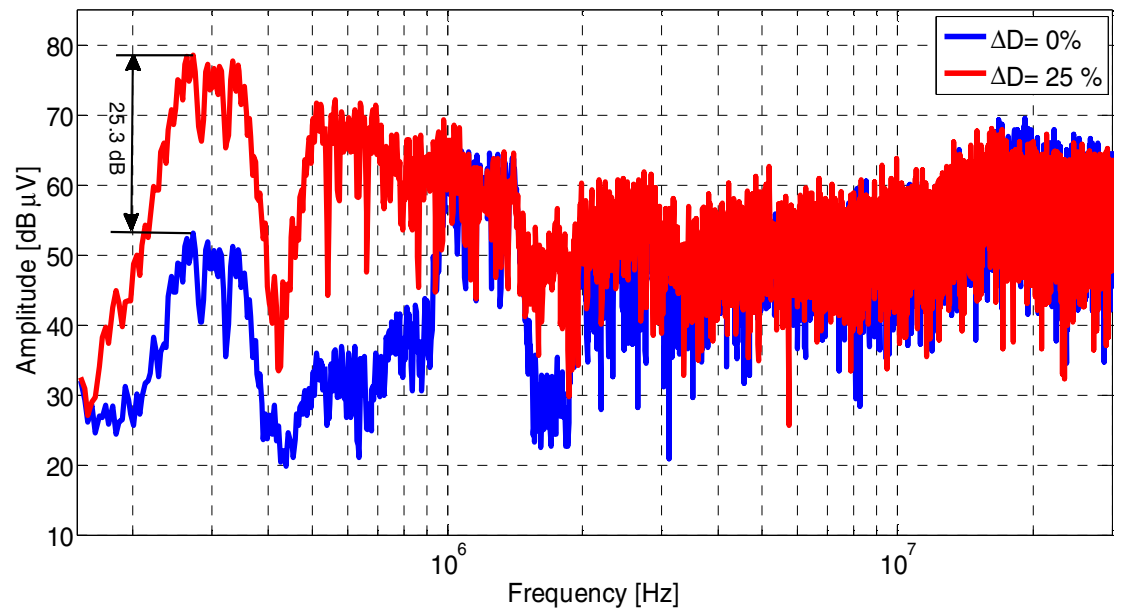

Fig. 8. Conducted EMI for $\Delta D=25 \%$. (Blue trace: $\Delta D=0 \%$;Red trace: $\Delta D=25 \%$ )

\section{Conclusion}

In this paper the idea of applying VDFM to CIMPC topology has been presented. In previous works, it has been demonstrated that VDFM is a worthy noise suppression technique. It successfully combines interleaving and frequency modulation to provide good attenuation features. It can be applied with a minimum cost in terms of weight, price and additional hardware. On the other hand, CIMPC topology appears as a good candidate where VDFM could be applied. In a real implementation some constructive mismatch will lead to a duty cycle deviation among the switching signal of each particular switching cell. Therefore, the effective attenuation will be smaller than in the case of ideal VDFM. This fact results in a worse attenuation of conducted EMI. We have shown that the higher the number of phase the higher influence of the duty-cycle absolute value deviation on the attenuation. It is important to underline that results shown here corresponds to a "worst-case" scenario, in which all imperfections of the circuit are concentrated in a single phase. In other words, the attenuation degradation in a real application will be actually below the values presented in this paper. However, we have shown the relevance of manufacturing issues in the EMI performance of the system. In order to reduce the effect of dispersion in terms of stray impedances an even number of phases in CIMPC topology can be used. Other alternative consists in implementing the coupled inductance on the same PCB. 


\section{References}

[1] J. Mon, J. Gago, D. Gonzalez, J. Balcells, R. Fernandez, I. Gil, and P. Bogonez: Modulation technique to reduce EMI in power multiconverters, IEICE Electronics Express Vol. 6 no. 8, pp.511-515, April 2009.

[2] J. Mon, J. Gago, D. Gonzalez, J. Balcells, R. Fernandez, and I. Gil: A new switching frequency modulation scheme for EMI reduction in multiconverter topology, In Proc. EPE'09, 13th European Conference on Power Electronics and Applications, pp.1-8, 2009.

[3] J.Mon, J. Gago, D. González, J. Balcells, R. Fernández, I. Gil, and P. Bogónez: EMI reduction by means of Switching Frequency Modulation with variable delay in power supplies, International Journal of Electronics Vol. 99 no. 1, pp.103-112, January 2012.

[4] J. Mon, D. González, J. Balcells, J. Gago, P. Bogónez: Hybrid Modulator for power converters in parallel topology, In Proc. EPE-PEMC 2012, 15th International Power Electronics and Motion Control Conference, 2012.

[5] F. Forest, T. A. Meynard, E. Labouré, V. Costan, E. Sarraute, A. Cuniere, T. Martire: Optimization of the Supply Voltage System in Interleaved Converters Using Intercell Transformers, IEEE Transactions on Power Electronics Vol. 22 no.3, pp.934-942, May 2007.

[6] B. Cougo, V. Costan, T. Meynard, F. Forest, E. Laboure: A new intercell transformer for interleaved converters, In Proc. EPE'09. Proc. EPE'09, 13th European Conference on Power Electronics and Applications, pp. 1-10, 2009.

[7] A.V. Peterchev, Jinwen Xiao, S.R. Sanders: Architecture and IC implementation of a digital VRM controller, IEEE Transactions on Power Electronics Vol. 18 no.1, pp. 356- 364, Jan 2003.

[8] F. Adam: Onduleur multicellulaire parallèle à ICT: commande équilibrante, analyse, modélisation, et optimisation des performances CEM, PhD Thesis, École Normale Superieure de Cachan, 2011.

[9] Suyong Chae; Yujin Song; Sukin Park; Hakgeun Jeong: Digital Current Sharing Method for Parallel Interleaved DC-DC Converters Using Input Ripple Voltage, IEEE Transactions on Industrial Informatics, Vol. 8 no.3, pp.536,544, Aug. 2012

[10] P. Zumel, O. Garcia, J. A. Oliver, and J. A. Cobos: Differential-Mode EMI Reduction in a Multiphase DCM Flyback Converter, IEEE Transactions on Power Electronics Vol. 24 no. 8, pp. 2013-2020, August 2009. 